\title{
Editorial: Special issue on agricultural robotics
}

\author{
Denny Oetomo $\cdot$ John Billingsley
}

Published online: 22 September 2010

(C) Springer-Verlag 2010

It can be argued that one of the earliest forms of robotics is agricultural automation, now undergoing a great revival in its activities. Its importance in the productivity of the agricultural crops is well established, but recent advancements in agricultural robotics seek not only to improve farming capacity with minimal human interference, but also to improve the efficiency of the tasks and the quality of the resulting crops through greater precision of the handling of the agricultural processes. This has seen the development in agricultural robotics centred around two main key phrases: autonomous agriculture and precision agriculture. The former is aimed at developing a capability to operate large farming facilities with minimum human manpower, such as by the development of autonomous tractors or coordinated teams of tractors and sensor networks to monitor a large farming area. The latter aims at developing precise data-gathering as well as crop or produce handling, such as selective spraying on plants as opposed to the traditional method of non-selectively spraying the entire field. Obviously, many applications also fall within both key phrases.

This special issue of Springer Journal of Intelligent Service Robotics on Agricultural Robotics seeks to present the latest developments in this exciting field of robotics research. The agricultural environment today serves not only as a client to which existing fundamental techniques of robotics are applied, but also as the source of challenges to the invention

D. Oetomo $(\bowtie)$

Department of Mechanical Engineering,

The University of Melbourne, Parkville, VIC 3010, Australia

e-mail: doetomo@unimelb.edu.au

J. Billingsley

Faculty of Engineering and Surveying,

University of Southern Queensland, Toowoomba, QLD 4350, Australia of new robotics strategies through its unique characteristics and requirements.

This special issue contains five peer-reviewed papers covering the exciting activities in the field of robotics applied in the agricultural environments. In McCarthy, Hancock, and Raine, a comprehensive review is presented for the use of automated visual inspection of plant conditions and its role in the variable rate irrigation and 'fertigation' of agricultural facilities. Correll et al. reported an exciting and unique indoor gardening project which would be autonomously tended by robotic agents that monitor the plant conditions and provide appropriate assistance. The paper presents details of the overall concept, the hardware implementation, as well as the preliminary results of the implementation. Berenstein, Shahar, Shapiro, and Edan investigate the potential of pesticide and hormone reduction in precision agriculture. In this paper, the authors have chosen to focus on the autonomous spraying of foliage and present four machine vision algorithms that facilitate selective spraying. The paper by Singh et al. presents an informative report on an ambitious project to integrate robotics and plant science, and to look into the socio-economic factors that could see robotics technology truly accepted into the main stream of agricultural technology. The paper reports on the lessons learned in the first year of the project, then focuses on several capabilities that have been integrated to be performed autonomously in an agricultural environment. The final paper in this special issue, by Zhang, Geimer, Noack and Grandl, presents an intelligent master-slave strategy between agricultural mobile machineries, with the strategy analysed in simulations and validated in field tests. Practical details of the implementation are discussed, highlighting the uncertainties encountered when systems are tested in the real field.

The guest editors would like to thank the authors, reviewers and the editorial team for their contributions and services 
to the special issue. We would also like to thank the Editorin-Chief, Professor Yuh, for his support and advice in the review and editorial process. We hope that the special issue will serve as a good reference in capturing the state-of-thearts in agricultural robotics today. 